\title{
Ausgezeichnet mit dem NPO-Label für Management Excellence
}

Christoph Gitz

Geschäftsführer SGPP

Korrespondenz:

SGPP/FMPP

Sekretariat

Postgasse 17

CH-3000 Bern 8

ines.frutig[at]psychiatrie.ch
Anlässlich der letzten Delegiertenversammlung Ende Oktober konnten die Verantwortlichen der Schweizerischen Gesellschaft für Psychiatrie und Psychotherapie SGPP das Zertifikat des NPO-Labels für Management Excellence entgegennehmen; Lohn für einen mehrjährigen kontinuierlichen Entwicklungsprozess ihrer Fachgesellschaft.

Was den Mitgliedern der SGPP an Qualität in ihrem beruflichen Alltag recht ist, muss der Fachgesellschaft billig sein. Unter diesem Motto und vor dem Hintergrund der sich abzeichnenden Wechsel im Präsidium und in der Sekretariatsleitung an der Geschäftsstelle machten sich die Verantwortlichen der SGPP vor gut zwei Jahren daran, die für das Funktionieren der Fachgesellschaft wichtigsten Prozesse und Dokumente zu skizzieren bzw. systematisch zu sammeln sowie für alle Mitarbeitenden im Sinne einer Wissenssicherung online verfügbar zu machen. rungsprozesses wurde auch deutlich, wo noch Nachholbedarf besteht und welche Projekte noch vertieft anzugehen sind.

Das NPO-Label für Management Excellence ist ein speziell für Verbände/Gesellschaften geschaffenes Label des VMI (Verbandsmanagement-Institut der Universität Fribourg) und der SQS (Schweizerische Vereinigung für Qualitäts- und Management-Systeme). Das über die reine ISO-Zertifizierung hinausgehende Label wird nur jenen Verbänden und Non-Profit-Organisationen verliehen, die in einem festgelegten und nachgewiesenen Masse ihre Führung nach dem heute verfügbaren Management-Wissen organisiert und implementiert haben. Anhand einer spezifischen Checkliste wird das Management-System der Organisation von besonders geschulten Assessoren geprüft. Diese stellen den Antrag auf die Verleihung des Labels, das offiziell von einem von VMI und SQS

\section{«Was den Mitgliedern der SGPP an Qualität in ihrem beruflichen Alltag recht ist, muss der Fachgesellschaft billig sein.»}

Da die gesicherten Prozesse neuen Mitarbeitenden und Vorstandsmitgliedern ihre Einarbeitung vereinfachen sollen, dient die Dokumentensammlung dazu, die tägliche Aufgabenverrichtung mit entsprechenden Vorlagen, Mustern zu erleichtern. Über Internet haben die Verantwortlichen in geschlossenen Benutzergruppen wie Kommissionen, Arbeitsund Projektgruppen zeitlich unbeschränkten Zugriff auf die jeweils aktuellste Version der für sie relevanten Prozesse und die damit verknüpften Dokumente. Selbstverständlich sind die zu erwartenden Arbeitserleichterungen für die Mitarbeitenden auf der Geschäftsstelle am höchsten. Während des Zertifizie- paritätisch zusammengesetzten Ausschuss verliehen wird.

Quasi zur Stabsübergabe des Präsidiums durften an der letzten Delegiertenversammlung vom 29. Oktober Hans Kurt als scheidender Präsident der SGPP und der neue Präsident Pierre Vallon vom Lead-Assessor der SQS, Roland Zürcher, das Zertifikat des aufgrund des Zertifizierungsassessments vom 10./11. August verliehenen NPO-Labels entgegennehmen. Mit kurzen jährlichen Erhaltungsaudits werden im Sinne eines kontinuierlichen Verbesserungsprozesses kleinere Optimierungsmassnahmen definiert und umgesetzt. Eine Rezertifizierung findet alle drei Jahre statt. 